\title{
Approximate Numerical Solution of Singular Integrals and Singular Initial Value Problems
}

\author{
Md. Habibur Rahaman ${ }^{1,}$, , M. Kamrul Hasan ${ }^{2}$, Md. Ayub Ali ${ }^{1}$, Md. Shamsul Alam² \\ ${ }^{1}$ Department of Mathematics, Jagannath University, Dhaka, Bangladesh \\ ${ }^{2}$ Department of Mathematics, Rajshahi University of Engineering \& Technology, Rajshahi, Bangladesh \\ Email address: \\ habiburhpr@gmail.com (M .H. Rahaman),mkh2502@yahoo.com (M. K. Hasan), ayub_ju@yahoo.com (M. A. Ali), \\ msalam1964@yahoo.com (M. S. Alam) \\ ${ }^{*}$ Corresponding author
}

\section{To cite this article:}

M. Habibur Rahaman, M. Kamrul Hasan, M. Ayub Ali, M. Shamsul Alam. Approximate Numerical Solution of Singular Integrals and Singular Initial Value Problems. American Journal of Applied Mathematics. Vol. 8, No. 5, 2020, pp. 265-270.

doi: $10.11648 /$ j.ajam.20200805.14

Received: August 6, 2020; Accepted: August 27, 2020; Published: September 21, 2020

\begin{abstract}
Numerical integration is one of the important branch of mathematics. Singular integrals arises in different applications in applied and engineering mathematics. The evaluation of singular integrals is one of the most challenging jobs. Earlier different techniques were developed for evaluating such integrals, but these were not straightforward. Recently various order straightforward formulae have been developed for evaluating such integrals but; all these integral formulae depend on Romberg technique for more accurate results. Based on these integral formulae, different order (up to fifth) implicit methods have been developed for solving singular initial value problems. These implicit methods give better results than those obtained by implicit Runge-Kutta methods but; the derivation of such higher order formulae are not so easy. In this article, a new third order straightforward integral formula has been proposed for evaluating singular integrals. This new formula is able to evaluate more efficiently than others existing formulae, moreover it has the independent ability to calculate very near accurate result to the exact value of the numerical integrals. Based on this new integral formula a new third order implicit method has been proposed for solving singular initial value problems. The new method provides significantly better results than other existing methods.
\end{abstract}

Keywords: Singular Integrals, Romberg Scheme, Singular Initial Value Problems, Implicit Runge-Kutta Methods

\section{Introduction}

Newton-Cotes formulae are worldwide used tools for evaluating numerical integrals; but cannot be used directly for singular cases [1]. Gauss's quadrature formulae and its extended formulae are used to evaluate such singular integrals [2]. Earlier, Fox used extrapolation technique for evaluating these integrals by Trapezoidal and Simpson's rules [3]. Fox also established a formula (called $U$ formula) for evaluating these integrals [3]. Later than, Huq et al. [4], Hasan et al. [5] and Rahaman et al. $[6,7]$ derive different order straightforward formulae for evaluating such type of singular integrals; but most of the formulae used Romberg scheme for better accuracy.

The studies of singular initial value problems have concerned the interest of many mathematicians and physicists in the recent years. First order singular initial value problems are encountered in ecology in the computation of avalanche run-up [8]. For the numerical solution of first order singular initial value problems, various schemes have been applied. Auzinger et al. [9] and Koch et al. [10, 11] applied well-known acceleration technique Iterated Defect Correction (IDeC) based on implicit Euler method to obtain high accuracy. Recently, Hasan et al. [12-16] derived second and third order implicit formulae based on the integral formulae for solving initial value problems having an initial singularity. Most Recently, Rahaman et al. [17] derived the third, fourth and fifth order implicit methods for solving initial value problems having initial singular point based on integral formulae. These implicit formulae give more accurate results than those obtained by the implicit Euler and 
implicit Runge-Kutta (RK2, RK3 and RK4) methods for first order singular initial value problems. In this article, a new third order integral formula has been proposed for evaluating singular integrals by introducing a small parameter $\mathcal{E}$. Based on the integral formula a third order implicit formula has been derived for solving initial value problems.

\section{Methodology}

In this section, first we discuss some recent existing

$$
f(x)=\frac{\left(x-x_{1}\right)\left(x-x_{2}\right)}{\left(x_{0}-x_{1}\right)\left(x_{0}-x_{2}\right)} f_{0}+\frac{\left(x-x_{0}\right)\left(x-x_{2}\right)}{\left(x_{1}-x_{0}\right)\left(x_{1}-x_{2}\right)} f_{1}+\frac{\left(x-x_{0}\right)\left(x-x_{1}\right)}{\left(x_{2}-x_{0}\right)\left(x_{2}-x_{1}\right)} f_{2}
$$

Considering $x_{1}=x_{0}+h / 3$ and $x_{2}=x_{0}+h$ and integrating Eq. (1) within limits $x_{0}$ to $x_{2}$, obtained the integral formula as

$$
\int_{x_{0}}^{x_{2}} f(x) d x=h\left(3 f_{1}+f_{2}\right) / 4
$$

Based on formula (2), Hasan et al. [13] derived second order implicit formula as

$$
y_{i+1}=y_{i}+h\left[3 f\left(x_{i}+h / 3,\left(y_{i}+\left(y_{i+1}-y_{i}\right) / 3\right)\right)+f\left(x_{i}+h, y_{i+1}\right)\right] / 4,
$$

where $i=0,1,2, .$. for solving initial value problems

$$
y^{\prime}(x)=f(x, y), \quad y\left(x_{0}\right)=y_{0}
$$

having an initial singular point of $f(x, y)$ at $x=x_{0}$.

Later Hasan et al. [5] derived another integral formula as

$$
\int_{x_{0}}^{x_{3}} f(x) d x=h\left(4 f_{1}+5 f_{2}+f_{3}\right) / 10
$$

by choosing $x_{1}=x_{0}+h / 6, x_{2}=x_{0}+4 h / 6$ and $x_{3}=x_{0}+h$. Based on formula (5), Hasan et al. [16] derived the third order implicit formula for solving singular initial value problems having singularity at $x=x_{0}$ as

$$
y_{i+1}=y_{i}+h\left[4 f\left(x_{i}+h / 6,\left(y_{i}+\left(y_{i+1}-y_{i}\right) / 6\right)\right)+5 f\left(x_{i}+4 h / 6,\left(y_{i}+4\left(y_{i+1}-y_{i}\right) / 6\right)\right)+f\left(x_{i}+h, y_{i+1}\right)\right] / 10 \text {, }
$$

where $i=0,1,2, \ldots$.

In the very recent time, by choosing $x_{1}=x_{0}+p h$, $x_{2}=x_{0}+q h \quad, \quad x_{3}=x_{0}+h \quad ; \quad p=(4-\sqrt{6}) / 10$, $q=(4+\sqrt{6}) / 10, w_{1}=(16-\sqrt{6}), w_{2}=(16+\sqrt{6}), w_{3}=4$ and using fourth order Lagrange's interpolation formula, Rahaman et al. [17] has been derive an integral formula as

$$
\int_{x_{0}}^{x_{3}} y(x) d x=h\left[w_{1} f_{1}+w_{2} f_{2}+w_{3} f_{3}\right] / 36
$$

where $f_{1}, f_{2}$ and $f_{3}$ are functional values of $f(x)$ respectively for $x_{1}=x_{0}+p h, x_{2}=x_{0}+q h$ and $x_{3}=x_{0}+h$. Based on formula (7) he presented a third order implicit formula for solving initial value problems as

$$
y_{i+1}=y_{i}+h\left[w_{1} f\left(x_{i}+p h, y_{i}+p\left(y_{i+1}-y_{i}\right)\right)+w_{2} f\left(x_{i}+q h, y_{i}+q\left(y_{i+1}-y_{i}\right)\right)+w_{3} f\left(x_{i}+h, y_{i+1}\right)\right] / 36,
$$

where $i=0,1,2, \ldots$

Also utilizing the fifth order Lagrange's interpolation formula, Rahaman et al. [17] derived the fourth order integral formula as 


$$
\text { choosing } \quad x_{1}=x_{0}+p h \quad, \quad x_{2}=x_{0}+q h \quad, \quad x_{3}=x_{0}+r h \quad, \quad w_{3}=0.32884431998006125
$$


different small values depending on the nature of functions it gives very near accurate results to the exact solutions of numerical integrals.

Table 1. Approximate values and absolute errors of the integral (19) by existing methods and proposed third order formula (17) for $\varepsilon=0.05$.

\begin{tabular}{lllll}
\hline Formulas & $\begin{array}{l}\text { Used } \\
\text { ordinates }\end{array}$ & Approx. Value & Abs. Error & Exact \\
\hline Huq_2 & 16 & 1.8396167933 & 0.1603832067 & \\
Hasan_3rd & 12 & 1.8460350986 & 0.1539649014 & \\
Rahaman_3rd & 12 & 1.8525557599 & 0.1474442401 & \\
Rahaman_4th & 12 & 1.8732054149 & 0.1267945850 & 2 \\
Rahaman_5th & 10 & 1.8761623901 & 0.1238376098 & \\
Gauss-Legender & 10 & 1.9170639420 & 0.0829360580 & \\
Prroposed_3rd & 3 & 1.9641113658 & 0.0358886342 & \\
\hline
\end{tabular}

\section{Examples}

In this section, firstly, some singular integral have been evaluated by proposed integral formula Eq. (17) and compare the results among Huq's (i.e .Eq. (2)), Hasan's (i.e. Eq. (5)), Rahaman's third (i.e. Eq. (7)), fourth (i.e. Eq. (9)), fifth (i.e. Eq. (11)) order integral formulae and Gauss quadrature formula. Then Romberg scheme and choosing optimum value of $\varepsilon$, very near accurate results to the exact values of some integrals have been presented. Secondly, some first order singular initial value problems have been solved by the proposed implicit formula (i.e. Eq. (18)) and the compared absolute errors among Hasan's third (i.e. Eq. (6)) and Rahaman's third (i.e. Eq. (8)), Rahaman's fourth (i.e. Eq. (10)) and Rahaman's fifth order (i.e. Eq. (12)) implicit formulae has been presented in graphs.

Example 3.1: Consider a singular integral in the form as

$$
I=\int_{0}^{1} \frac{1}{\sqrt{x}} d x
$$

Table 2. The Romberg scheme of the integral (19) by the proposed formula (17) for $\varepsilon=0.05$

$\begin{aligned} & N(h)=1.9641113657918943 \\ & N\left(\frac{h}{2}\right)=1.9744124023521268\end{aligned} \quad N\left(h, \frac{h}{2}\right)=1.999281304522341$

Example 3.2: Consider another stronger singular integral in the form as

$$
I=\int_{0}^{1} \frac{e^{-x}}{x^{\frac{3}{4}}} d x
$$

Table 3. Approximate values and absolute errors of the integral (20) by existing methods and proposed formula (17) for $\varepsilon=0.05$.

\begin{tabular}{llll}
\hline Formulas & Used ordinates & Approx. Value & Error \\
\hline Huq_2nd & 16 & 2.1618969054 & 1.2174574736 \\
Hasan_3rd & 12 & 2.1837126223 & 1.1956417567 \\
Rahaman_3rd & 12 & 2.2089397760 & 1.1704146029 \\
Rahaman_4th & 12 & 2.2934285211 & 1.0859258578 \\
Rahaman_5th & 10 & 2.3058271615 & 1.0735272174 \\
Gauss-Legender & 10 & 2.5007326738 & 0.8786217052 \\
Proposed_3rd & 3 & 2.5398969989 & 0.8394573801 \\
\hline
\end{tabular}

Table 4. The Romberg scheme of the integral (20) by the proposed formula (17) for $\varepsilon=0.05$.

\begin{tabular}{ll}
\hline$N(h)=2.53989699891691$ & $N\left(h, \frac{h}{2}\right)=3.36305183100028$ \\
$N\left(\frac{h}{2}\right)=2.67086388350260$ & \\
\hline
\end{tabular}

\begin{tabular}{|c|c|c|c|c|}
\hline Problems & Optimum values of $\varepsilon$ & Exact value & Approx. value & Error \\
\hline $\int_{0}^{1} \frac{d x}{\sqrt{x}}$ & 0.045291458012 & 2 & 1.9999999999987602 & $1.2399 \times 10^{-12}$ \\
\hline $\int_{0}^{1} \frac{e^{-x} d x}{x^{\frac{3}{4}}}$ & 0.028278057125 & 3.3793543790284106 & 3.3793543790229128 & $5.49782 \times 10^{-12}$ \\
\hline $\int_{0}^{1} \sqrt{x} d x$ & 0.0633974569 & $\frac{2}{3}$ & 0.6666666666665948 & $7.18314 \times 10^{-14}$ \\
\hline$-\int_{0}^{1} x \log x d x$ & 0.07340721 & $\frac{1}{4}$ & 0.2499999999003209 & $9.96791 \times 10^{-11}$ \\
\hline$-\int_{0}^{1} \frac{x \log x d x}{1+x}$ & 0.087639324981 & $\begin{array}{c}1-\pi^{2} / 12= \\
0.1775329665758869\end{array}$ & 0.17753296657884635 & $2.95938 \times 10^{-12}$ \\
\hline
\end{tabular}

Table 5. Approximate values of the different integrals by proposed integral formula (17) for proper chose or optimum values of $\mathcal{E}$. 


\begin{tabular}{|c|c|c|c|c|}
\hline Problems & Optimum values of $\varepsilon$ & Exact value & Approx. value & Error \\
\hline$-\int_{0} \sqrt{x} \log x d x$ & 0.0801627431 & $\frac{4}{9}$ & 0.44444444444468951 & $2.45071 \times 10^{-12}$ \\
\hline$-\int_{0}^{\log x} \frac{1}{\sqrt{x}} d x$ & 0.036523596871863 & 4 & 3.9999999999999943 & $5.68434 \times 10^{-14}$ \\
\hline $\int_{0}^{1} e^{-x^{2}} d x$ & 0.0498246631 & 0.746824132812427 & 0.7468241328126574 & $2.30371 \times 10^{-13}$ \\
\hline $\int_{0}^{\pi} x^{\pi} \operatorname{Sin} \sqrt{x} d x$ & 0.226804165965 & 27.255515984803424 & 27.25551598480386 & $4.36984 \times 10^{-13}$ \\
\hline
\end{tabular}

Example 3.4: Consider the first order linear singular initial value problem as

$$
y^{\prime}(x)=-\frac{y(x)}{\sqrt{x}}, 0<x \leq 1, y(0)=1
$$

The exact solution of equation (21) is obtained as $e^{-2 \sqrt{x}}$. The absolute error of the solution of the equation (21) obtained by the Hasan's [13], Rahaman's [17] and the present method (18) for $\varepsilon=0.05$ are plotted in Figure-1 (a) for $h=0.001$ and in Figure-1 (b) for $h=0.0001$.

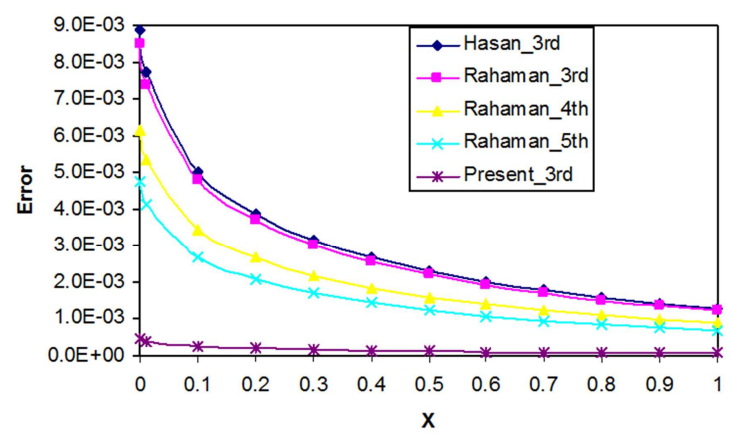

(a)

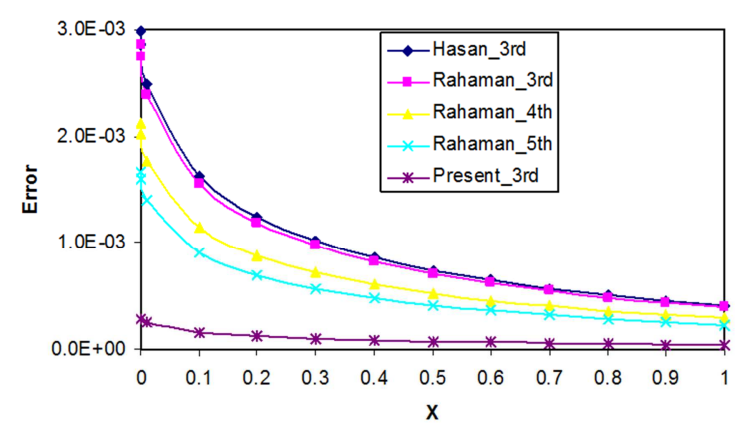

(b)

Figure 1. The absolute error of the Eq. (21) by different methods for $h=0.001$ in (a) and for $h=0.0001$ in (b).

Example 3.5: Considering a first order non-non linear singular initial value problem as

$$
y^{\prime}(x)=-\frac{y^{2}(x)}{\sqrt{x}}, 0<x \leq 1, y(0)=1
$$

The exact solution of equation (22) is obtained as $1 /(1+2 \sqrt{x})$. The absolute error of the solution of the equation (22) obtained by the Hasan's [13], Rahaman's [17] and the present method (18) for $\varepsilon=0.05$ are plotted in Figure-2 (a) for $h=0.001$ and in Figure-2 (b) for $h=0.0001$.

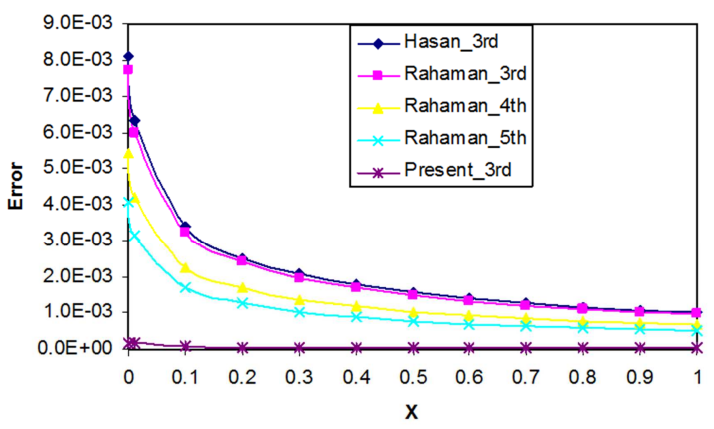

(a)

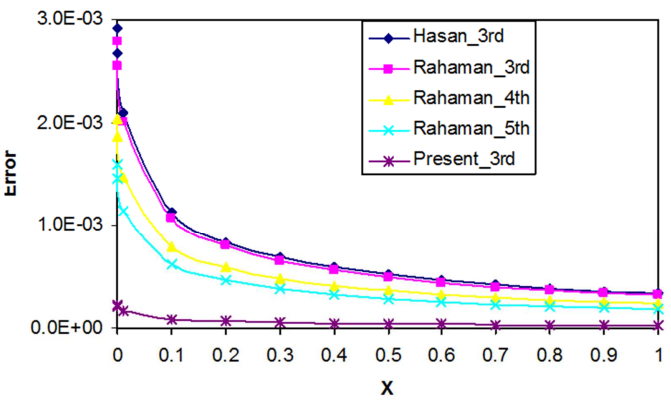

(b)

Figure 2. The absolute error of the Eq. (22) by different methods for $h=0.001$ in (a) and for $h=0.0001$ in $(b)$.

\section{Results and Discussion}

The results of singular integrals and singular initial value problems obtained by this proposed method has been compared with others existing methods. 
For the singular integral, in Table 1 and Table 3 it is observed that the proposed formula (17) gives significantly better result than existing formulae using fewer ordinates. In Table 2 and Table 4 it is shown that, the Romberg scheme of the proposed formula also converges. In Table 5 it is shown that, without the help of Romberg technique; the proposed formula (17) provides very near accurate results to the exact values of different integrals by proper (optimal) chose of $\varepsilon$ depending on the nature of functions.

Also, for first order initial value problems, in Figure 1 (a), Figure 1 (b), Figure 2 (a) and Figure 2 (b), it is observed that, the absolute error of solution of Eqs. (21) and (22) obtained by the proposed formula (18) is smaller than Hasan's [13] and Rahaman's [17] formulae.

\section{Conclusion}

From the above discussion it is observed that, the proposed third order formula provide significant better results than existing methods both for singular integrals and singular initial value problems. Moreover, the proposed integral formula provide very close results to exact values for optimal choice of parameter $\varepsilon$. So, it may conclude that the proposed third order formula may play an important role in the field of numerical integrations as well as singular initial value problems.

\section{References}

[1] S. S. Sastry, Introductory Methods of Numerical analysis, $2^{\text {nd }}$ Ed., Prentice-Hall of India Private Lim., New Delhi, 1995.

[2] M. K. Jain, S. R. K. Lyengar and R. K. Jain, Numerical Methods for Scientific and Engineering Computation, Second Edition, Wiley Eastern Limited, New Delhi, pp 219-227. ISBN-0-85226-434-8.

[3] L. Fox, Romberg integration for a class of singular integrand, Comput. J. 10 (1) (1967), pp 87-93.

[4] M. Ashraful Huq, M. Kamru Hasan, M. Majedur Rahman, M. Shamsul Alam, A simple and straightforward method for evaluating some singular integrals, Far East Journal of Mathematical Education. 7 (2), 2011, pp. 93-103.

[5] M. Kamrul Hasan, M. Ashraful Huq, M. Habibur Rahaman, B.M. Ikramul Haque, A More Accurate and Straightforward Method for Evaluating Singular Integrals, Universal Journal of Applied Mathematics 3 (3): pp. 53-61, 2015.

[6] Md. Habibur Rahaman, Md. Ashraful Huq, M. Kamrul Hasan,
A New Straightforward Method for Evaluating Singular Integrals", Applied and Computational Mathematics. 4 (6), pp. 420-423, 2015.

[7] Md. Habibur Rahaman, M. Kamrul Hasan, Md. Ashraful Huq, Md. Suzan Ahamed and M. Shamsul Alam, A higher order straightforward method for evaluating singular integrals, Journal of Calcutta Mathematical Society, 11 (1), pp. 11-16, 2015.

[8] S. Chandra sekhar, Introduction to the study of stellar structure. Dover: New York 1967.

[9] W. Auzinger, O. Koch, P. Kofler and E. Weinmuller (2000): Acceleration techniques for solution of initial value problems, Project report, No. 129/00, Department of Applied Mathematics and Numerical Analysis, Vienna University of Technology, Austria.

[10] O. Koch, P. Kofler, E. Weinmuller, The Implicit Euler method for the numerical solution of singular initial value problems. Appl Num Math 2000; 34: pp. 231-252.

[11] O. Koch, E.Weinmuller, Analytic and numerical treatment of a singular initial value problem in avalanche modeling. Appl Math Comput 2004; 148: pp. 561-570.

[12] M. Kamrul Hasan, M. A Huq, M. Shaifur Rahman, M. M. Rahman, M. S Alam. A new implicit method for numerical solution of singular initial value problems. International Journal of Conceptions on Computing and Info Technology 2014; 2 (1): pp. 87-91.

[13] M. Kamrul Hasan, M. Suzan Ahamed, M. S. Alam, M. B. Hossain. An implicit method for numerical solution of singular and stiff initial value problems. J Comput Eng 2013; Article ID 720812.

[14] M. Kamrul Hasan, M. Suzan Ahamed, M. S. Alam, M. B. Hossain. An implicit method for numerical solution of second order singular initial value problems. The open Math J. 2014; 7: pp 1-5.

[15] M. Kamrul Hasan, M. Suzan Ahamed. An implicit method for numerical solution of system of first-order singular initial value problems. Journal of Advances in Mathematics and Computer Science 2018; 27 (2): pp 1-11.

[16] M. Kamrul Hasan, M. Suzan Ahamed, B. M. Iqramul Haque, M. S. Alam, M. B. Hossain. A higher order implicit method for numerical solution of singular initial value problems. Communications in Computer and Information Science, 2017; 655: pp 255-264.

[17] Md. Habibur Rahaman, M. Kamrul Hasan, Md. Ayub Ali, and M. S. Alam. Implicit Methods for Numerical Solution of Singular Initial Value Problems. Applied Mathematics and Nonlinear Sciences (aop), 2020; pp 1-8. doi: 10.2478/AMNS.2020.2.00001. 